\title{
Retinal dystrophies associated with peripheral retinal vasculopathy
}

\author{
W. S. GRIZZARD, A. F. DEUTMAN, AND A. J. L. G. PINCKERS \\ From the Institute of Ophthalmology, University of Nijmegen, The Netherlands
}

SUMMARY A pair of identical twins with cone-rod dystrophy and 1 patient with dominant retinitis pigmentosa are reported who had a peculiar vascular retinopathy characterised by perivenous accumulation of fluorescein in the temporal periphery. Other retinal dystrophies associated with leaking retinal vessels are discussed.

An association between retinal dystrophies and retinal vascular disease has been noted previously (Notting and Deutman, 1976). Retinitis pigmentosa associated with Coats's disease (Zamorani, 1956; Morgan and Crawford, 1968; Schmidt and Faulborn, 1970; Schmidt and Faulborn, 1972; Witschel, 1974; Lanier et al., 1976; Ayesh et al., 1976) and retinitis pigmentosa associated with macular oedema (Hyvärinen et al., 1971; Ffytche, 1972) are well known. Dominant pedigrees of cystoid macular oedema have been seen in association with an atrophic macular dystrophy (Deutman et al., 1976; Pinckers et al., 1976; Notting and Pinckers, 1977).

Peripheral leaking vessels have been reported in Favre-Goldmann syndrome (Fishman et al., 1976) and in sex-linked juvenile retinoschisis (Notting and Deutman, 1976). A case of Stargardt's macular dystrophy with Coats's disease (Deutman, 1975) and a patient with cone dystrophy with leaking capillaries in the posterior pole have been reported (Notting and Deutman, 1976). A dominantly inherited exudative vitreoretinopathy with fluorescein leakage predominantly involving temporal retinal vessels has also been described (Criswick and Schepens, 1969; Gow and Oliver, 1971; Canny and Oliver, 1976).

Recently we studied a pair of identical twins with a severe cone-rod dystrophy and a patient with dominant retinitis pigmentosa, who all showed a similar, bilateral, symmetrical, peripheral vasculopathy of the temporal retina. Fluorescein leakage was demonstrated in all 3 patients, but the miliary capillary aneurysms and exudative, bullous detachments typical of Coats's disease were not seen. To

Address for reprints: Dr A. F. Deutman, Institute of Ophthalmology, St. Radboud Hospital, Philips van Leydenlaan 15, Nijmegen, The Netherlands our knowledge the association of cone-rod dystrophy and retinitis pigmentosa with a peripheral vasculopathy of this type has not been reported previously.

\section{Methods}

Complete examination including Goldmann contact lens, fundus photography, fluorescein angiography, electroretinography, electro-oculography, and dark adaptometry was performed in Nijmegen. Examination and electroretinography had been previously performed in Rotterdam on 2 patients. These techniques have been described in detail elsewhere (Deutman, 1971; Thijssen et al., 1974). Colour vision was initially tested with the AO-HRR pseudoisochromatic plates. If unable to identify any AO-HRR plates, the patient was asked to identify the colour on large saturated panels of red, green, blue, and yellow. If the patient was able to perform the AO-HRR test, then a modified panel D-15 (using the new colour test of Lanthony, value/ chroma 6/4) and the 100-hue test were used to assess colour vision.

\section{Case reports}

(1) A 10-year-old male patient who resides in an institution for the partially sighted was referred to the university clinic in Nijmegen for evaluation. The patient had had progressive loss of vision since the age of 7. There was no photophobia, and the patient had shown no mental deterioration.

The visual acuity was counting fingers at $1 \mathrm{~m}$ in the right eye and counting fingers at $2 \mathrm{~m}$ in the left eye. Nystagmus was absent. The anterior chamber was normal. There were fine dust-like particles throughout the vitreous of both eyes. The patient had a marked, generalised, bilateral pigmentary 


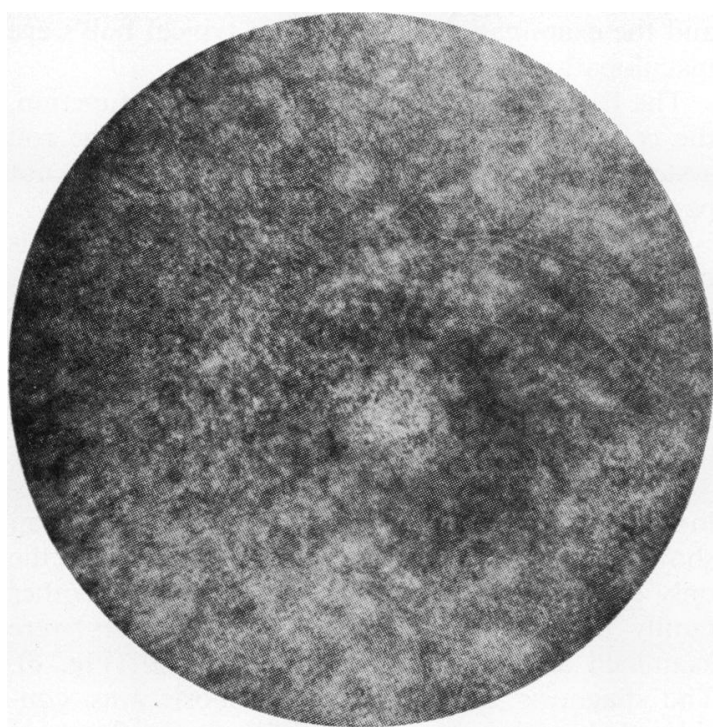

Fig. 1 Right eye, case 1. Fluorescein angiogram in prearterial phase shows fine pigment mottling of the pigment epithelium

retinopathy. The macula had a grey-white reflex with loss of pigment (Fig. 1). The choroidal vessels were not readily visible. The pigment was irregularly dispersed in a fine granular pattern throughout the periphery. The disc was waxy pale, and the vessels were markedly attenuated. No vascular abnormalities or exudates were seen.

Pregnancy had been uncomplicated, as was delivery at 8 months gestation. No maternal infections were noted during pregnancy. No oxygen was given at any time. The child's development had been slow, but progress had been stable. The patient had been studied in Rotterdam 2 years previously, and the examination had shown a typical bull's eye pattern in both maculas. At that time the ERG was sub-normal; the cone system was affected more than the rod system. The patient was unable to identify any symbols on the AO-HRR pseudoisochromatic plates, even missing the initial instruction plate. The patient was able to name correctly the colours on the large saturated panels. Fluorescein angiography in Nijmegen showed irregular loss of pigment and leaking temporal vessels in both eyes (Figs. 2 and 3). Fluorescein accumulated temporally in late stages of the angiogram. The ERG, done in 1976 in Nijmegen, was not recordable, and the EOG was flat. Dark adaptometry and visual fields were not performed.

(2) The twin brother of case 1 is also in an institution and had had loss of vision since the age of 7. No photophobia was evident during the

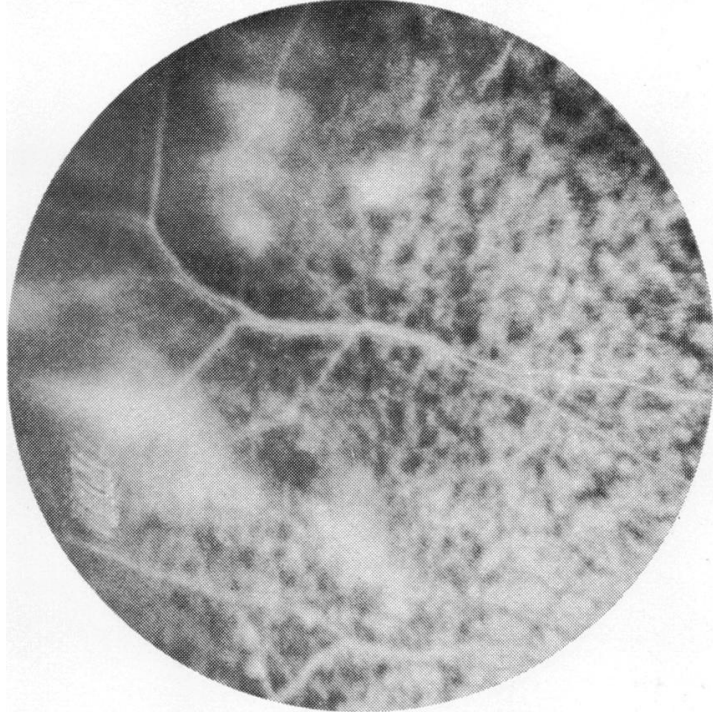

Fig. 2 Right eye, case 1. Late stage of fluorescein angiogram shows peripheral accumulation of fuorescein round the vessels in the temporal periphery

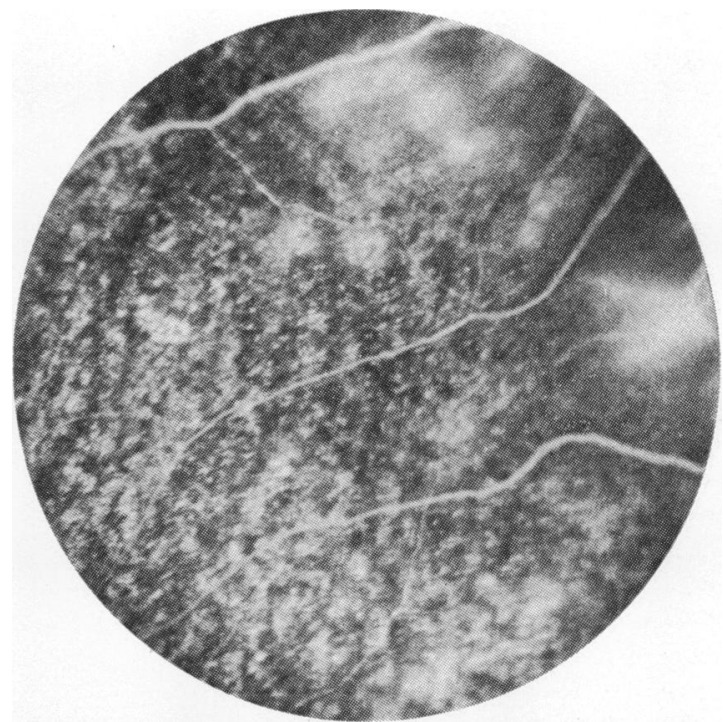

Fig. 3 Left eye, case 1. Late stage of fluorescein angiogram shows similar changes in the other eye of the same patient

examination. The visual acuity was counting fingers at $2 \mathrm{~m}$ in both eyes. The anterior chamber was normal. Small, dust-like particles were dispersed throughout the vitreous. The retina showed a finely granular pigment retinopathy (Fig. 4) with a beaten-bronze, yellowish macular reflex. Choroidal 


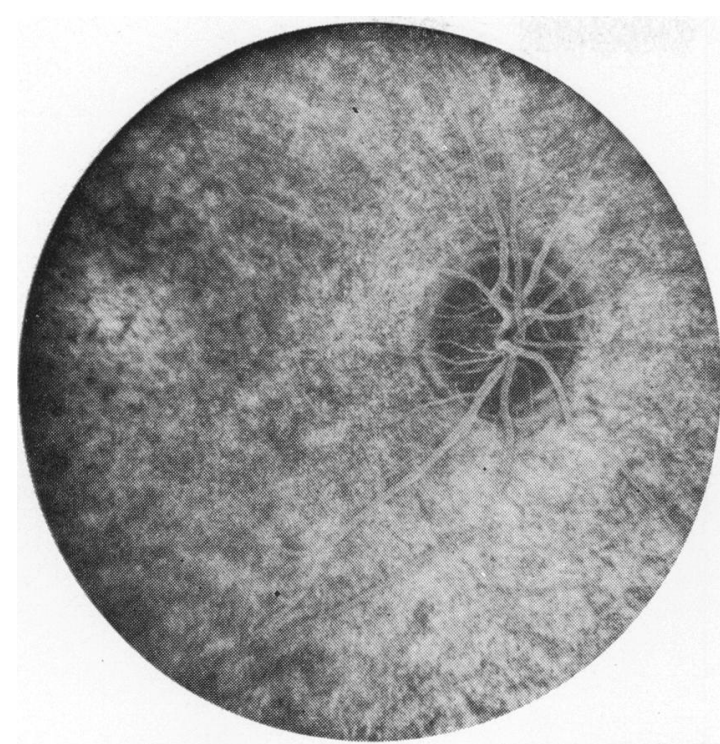

Fig. 4 Right eye, case 2. Late stage of angiogram shows macula with irregular pigmentation and faint remnants of bull's eye pattern brought out by fluorescein

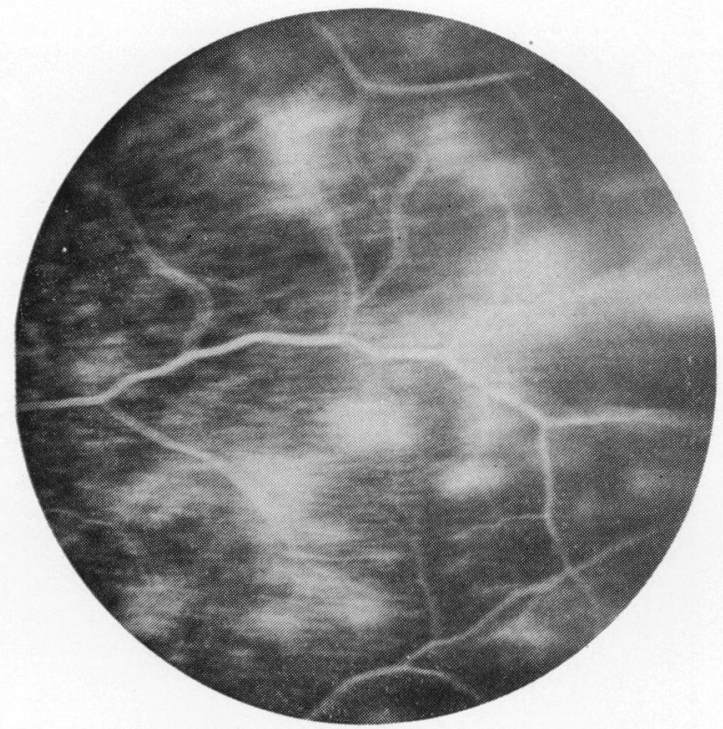

Fig. 5 Left eye, case 2. Late stage of angiogram shows accumulation of fluorescein along temporal vein. Suggestion of vessel staining can be seen superiorly

vasculature was not prominently visible. The disc was pale and the vessels were attenuated. Peripherally no vascular abnormalities were noted. The birth history was unremarkable and the child had received no neonatal oxygen. This patient also had been studied for two years previously in Rotterdam, and the examination had shown a typical bull's eye maculopathy.

The ERG had demonstrated subnormal function, the cone system being more affected than the rod system. Dark adaptometry and perimetry were not performed.

The patient was unable to see any of the symbols on the AO-HRR pseudoisochromatic plates, but could identify correctly the large saturated colour panels. Fluorescein angiography showed a finely granular pigment retinopathy and bilateral temporal leaking and staining vessels with accumulation of fluorescein in the late stages (Fig. 5).

Neither patient had any other medical or neurological problems, and an electroencephalogram showed normal cortical activity. The twins were the only children of the marriage. There was no other family history of eye diseases. The parents were examined and were found to be normal (Fig. 6). The diagnosis of ceroid lipofuscinosis was considered, but since no abnormality in development had been noted this was thought to be unlikely.

(3) A 35-year-old white male was examined as part of a family study of dominant retinitis pigmentosa. He had no complaints of night blindness,
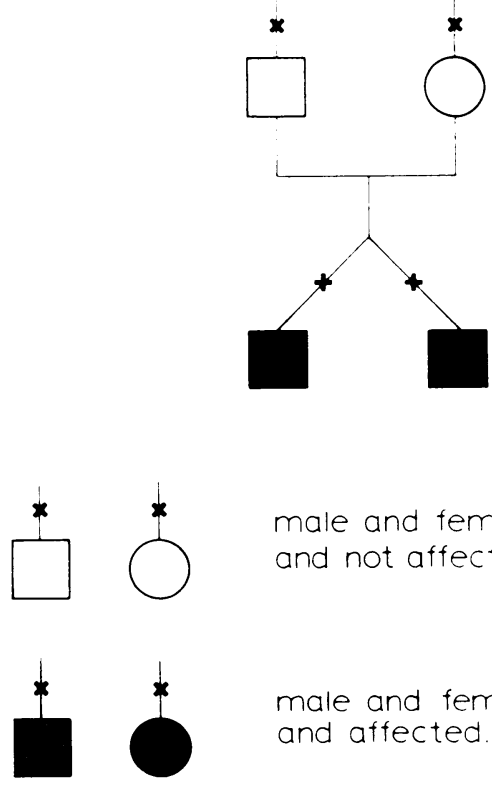

male and female; examined and affected.

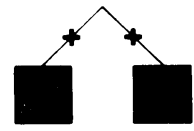

twins, examined and affected.

Fig. 6 Pedigree of cases 1 and 2. Both identical twins were affected. The parents were normal 
though 3 of 12 siblings had this complaint. Visual acuity was 0.8 in each eye. The anterior chamber and the vitreous were normal. The fundus showed a tapetal reflex with attenuated vessels and a slightly pale optic disc.

The ERG showed a subnormal response with the rod system affected more than the cone system. The EOG was flat. Dark adaptometry was moderately subnormal. The modified D-15 test showed one minor error in the left eye and the 100-hue test showed 81 errors in the right eye and 118 errors in the left eye. No definite axis was noted. Fluorescein angiography showed leaking, staining vessels in the temporal periphery in both eyes (Figs. 7 and 8).

\section{Discussion}

Retinal dystrophies may be classified according to which photoreceptor is primarily involved. This may be measured objectively with electroretinopathy and electro-oculography and subjectively by colour vision testing and dark adaptometry.

Cone dystrophy is a general, functional classification which includes all the progressive inherited retinal degenerations showing predominant involvement of cone function. The cone dystrophies have been classified by Goodman et al. (1963), Krill et al. (1973), and by François et al. (1976). In all classifications patients with both cone and rod involvements were described.

Patients 1 and 2 have a cone-rod dystrophy in which the cone system is affected primarily and predominantly and the rod system is involved later in the degenerative process. The inheritance of cone dystrophy is autosomal dominant or autosomal recessive; sporadic cases have also been frequently reported (Goodman et al., 1963; Krill et al., 1973; François et al., 1976).

Retinitis pigmentosa is a general classification of retinal dystrophies in which the rod system is affected predominantly and primarily and the presenting symptoms of loss of peripheral vision and poor night vision reflect dysfunction of the rod system. Dominant, recessive, and sex-linked inheritance has been described; sporadic cases have also been frequently seen (Merin and Auerbach, 1976). The cone system may also come to be involved, and the distinction between retinitis pigmentosa and cone dystrophy may blur as both types of photoreceptors become affected.

Peripheral retinal vasculopathy of this type has not been reported before in cone dystrophy or retinitis pigmentosa to our knowledge. Notting and Deutman (1976) reported capillary leakage from inframacular capillaries in the posterior pole of 1 patient with a progressive cone dystrophy. That

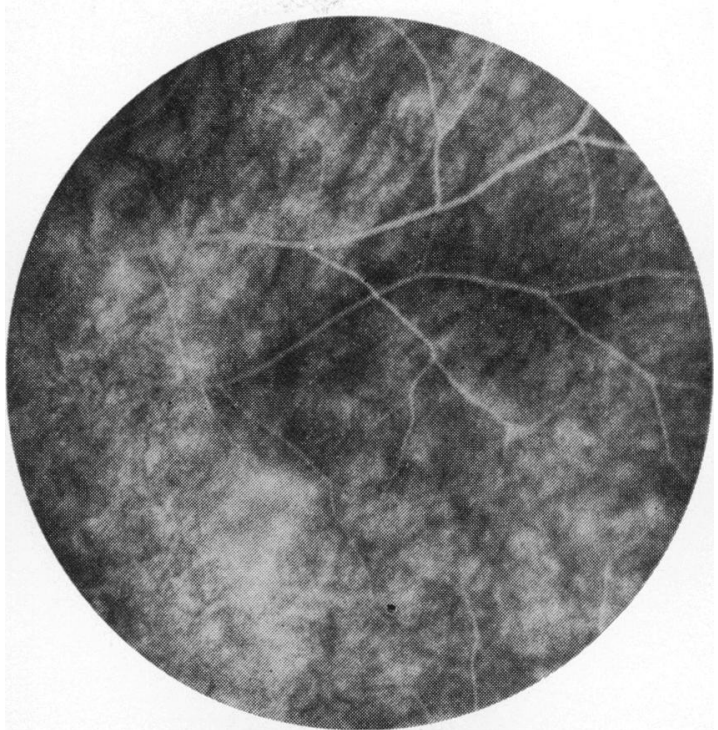

Fig. 7 Right eye, case 3. Late angiogram of temporal periphery shows staining of temporal veins and accumulation of fluorescein

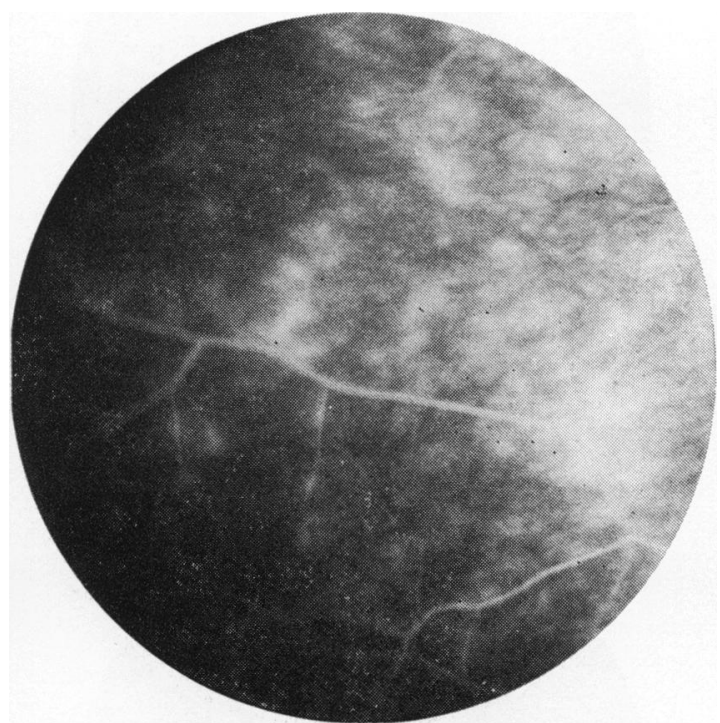

Fig. 8 Left eye, case 3. In late stage of angiogram there is definite venous staining with accumulation of fluorescein in several places

patient's younger sister also had a cone dystrophy and dilated capillaries, but no fluorescein leakage was seen. Diffuse, generalised permeability of retinal capillaries to fluorescein and cystoid macular oedema are well known in retinitis pigmentosa (Hyvärinen et al., 1971; Ffytche, 1972). 

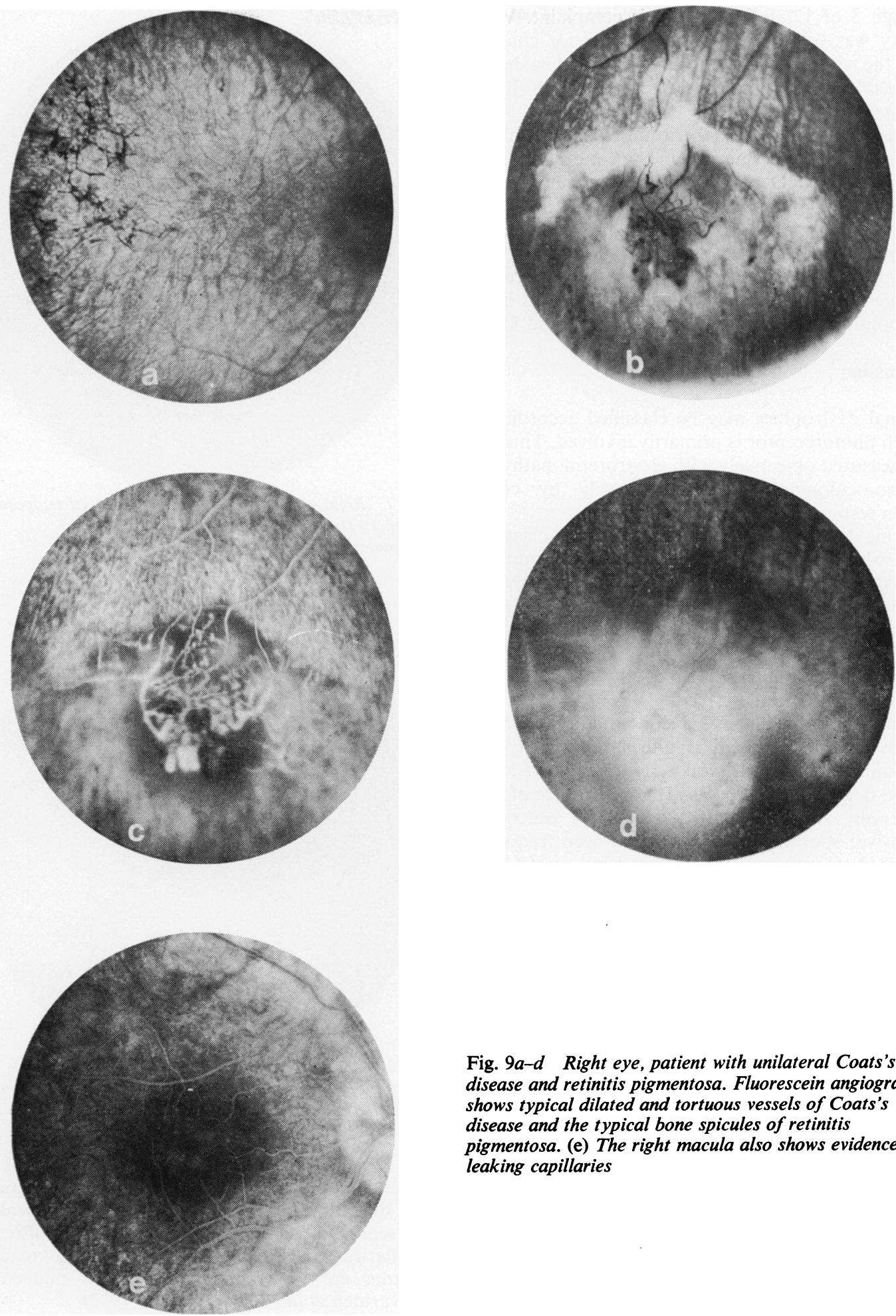

Fig. 9a-d Right eye, patient with unilateral Coats's disease and retinitis pigmentosa. Fluorescein angiogram shows typical dilated and tortuous vessels of Coats's disease and the typical bone spicules of retinitis pigmentosa. (e) The right macula also shows evidence of leaking capillaries 
Cases of Coats's disease have also been associated with retinitis pigmentosa. This form of Coats's disease is different, however, from the sporadic Coats's disease, which is seen mostly in young males. Every reported case of Coat's's disease associated with retinitis pigmentosa we reviewed in the literature was bilateral. One of us (A.D.) has, however, seen 2 cases of unilateral Coats's disease associated with retinitis pigmentosa (Fig. 9).

Five of the 11 reported cases were familial, and over half of the cases were female (Zamorani, 1956; Morgan and Crawford, 1968; Schmidt and Faulborn, 1970, 1972; Witschel, 1974; Lanier et al., 1976; Ayesh et al., 1976). Coats's disease is usually unilateral and non-familial (Woods and Duke, 1963; Morales, 1965; Chisholm et al., 1974; Spitznas et al., 1975).

One case of a unilateral Coats's disease has been reported associated with Stargardt's macular dystrophy (Deutman, 1976) and dominant cystoid macular oedema has been reported in which a dominant inherited macular dystrophy is associated with macular oedema and leaking macular capillaries (Deutman et al., 1976; Pinckers et al., 1976; Notting and Pinckers, 1977). One case of peripheral leaking veins and capillaries has also been reported in sex-linked retinoschisis (Notting and Deutman, 1976).

Dominant exudative vitreoretinopathy has been well documented. The temporal periphery is predominantly affected by an exudative retinopathy of unknown aetiology. Demarcation lines, nonperfusion on the far retinal periphery, retinal elevation, and vitreal abnormalities are characteristic of this disease (Criswick and Schepens, 1969; Gow and Oliver, 1971; Canny and Oliver, 1976). Abnormal electrophysiology in cases without retinal detachment has not been reported, and our experience confirms this.

In Favre-Goldmann syndrome 3 cases which showed peripheral leaking veins have been described (Fishman et al., 1976). Leaking of fluorescein was seen peripherally around occluded vessels in areas of retinoschisis, and the posterior pole showed generalised permeability to fluorescein from the retinal capillaries. Cystoid macular oedema was also present.

The fluorescein angiograms of our 3 patients were very similar to each other. The transmit time of the fluorescein was increased, and the temporal periphery showed venous dilatation, perivenous accumulation of fluorescein, and staining of the venous walls. The retinal vessels of the temporal periphery are also involved in the pathogenesis of retrolental fibroplasia (Cantolino et al., 1971; Payne and Patz, 1972). These temporal vessels are still forming at birth (Cogan, 1963) and are sensitive to the effects of oxygen. In our cases there were no demarcation lines, non-perfused areas, or retinal elevation characteristic of retrolental fibroplasia (Cantolino et al., 1971; Payne and Patz, 1972).

Witschel (1974) has suggested that irritating substances might be released by the degenerating retina in retinitis pigmentosa and that this process might cause vascular leakage and the clinical picture of Coats's disease. A similar toxic substance may account for the leaking capillaries seen in our patients with retinitis pigmentosa and cone dystrophy and also seen in other patients with inherited retinal dystrophies and leaking capillaries. This may be a direct effect on the retinal capillaries or an indirect effect via inflammatory mechanisms. We suspect the peripheral vascular leakage is rather common in severe retinal dystrophies but is not seen because the periphery is frequently not examined during fluorescein angiography.

We are grateful to $\mathrm{Mr}$ Albert Aan de Kerk for providing the photographs.

\section{References}

Ayesh, I., Sanders, M. D., and Friedmann, A. I. (1976). Retinitis pigmentosa and Coats's disease. British Journal of Ophthalmology, 60, 775-777.

Canny, C. L. B., and Oliver, G. L. (1976). Fluorescein angiographic findings in familial exudative vitreoretinopathy. Archives of Ophthalmology, 94, 1114-1120.

Cantolino, S. J., O'Grady, G. E., Herrera, J. A., Israel, C., Justice, J., and Flynn, J. T. (1971). Opthalmoscopic monitoring of oxygen therapy in premature infants; fluorescein angiography in acute retrolental fibroplasia. American Journal of Ophthalmology, 72, 322-331.

Chisholm, I. A., Foulds, W. S., and Christison, D. (1974). Investigation and therapy of Coats's disease. Transactions of the Ophthalmological Societies of the United Kingdom, 94, 335-341.

Cogan, D. G. (1963). Development and senescence of the human retinal vasculature. Transactions of the Ophthalmological Societies of the United Kingdom, 83, 465-489.

Criswick, V. G., and Schepens, C. K. (1969). Familial exudative vitreoretinopathy. American Journal of Ophthalmology, 68, 578-594.

Deutman, A. F. (1971). The Hereditary Dystrophies of the Posterior Pole of the Eye. Van Gorcum: Assen, The Netherlands.

Deutman, A. F. (1975). Unexpected findings in hereditary macular dystrophy. In New Developments in Ophthalmology, Documenta Ophthalmologica Proceedings Series, vol. 7, pp. 281-312. Ed. A. F. Deutman. Junk: The Hague.

Deutman, A. F., Pinckers A. J. L. G., and Aan de Kerk, A. L. (1976). Dominantly inherited cystoid macular edema. American Journal of Ophthalmology, 82, 540-548.

Fishman, G. A., Jampol, L. M., and Goldberg, M. F. (1976). Diagnostic features of the Favre-Goldmann syndrome. British Journal of Ophthalmology, 60, 345-353.

Ffytche. T. J. (1972). Cystoid maculopathy in retinitis pigmentosa. Transactions of the Ophthaimological Socteties of the United Kingdom, 92, 265-283.

François, J., De Rouck, A., and De Laey, J. J. (1976). 
Progressive cone dystrophies. Ophthalmologica, 173, 81-101. Goodman, G., Ripps, H., and Siegel, I. M. (1963). Cone dysfunction syndromes. Archives of Ophthalmology, 70, 214-231.

Gow, J., and Oliver G. L. (1971). Familial exudative vitreoretinoapthy. Archives of Ophthalmology, 86, 150-155.

Hyvärinen L., Maumenee, A. E., Kelly, J., and Cantolino, S. (1971). Fluorescein angiographic findings in retinitis pigmentosa. American Journal of Ophthalmology, 71, 17-26.

Krill, A. E., Deutman, A. F., and Fishman, M. (1973). The cone degenerations. Documenta Ophthalmologica, 35, 1-80.

Lanier J. D., McCrary, J. A., and Justice, J. (1976), Autosomal recessive retinitis pigmentosa and Coats's disease. Archives of Ophthalmology, 94, 1737-1742.

Merin, S., and Auerbach, E. (1976). Retinitis pigmentosa. Survey of Ophthalmology, 20, 303-346.

Morales, A. G. (1965). Coats's disease. Natural history and results of treatment. American Journal of Ophthalmology, 60, 855-865.

Morgan, W. E., and Crawford, J. B. (1968). Retinitis pigmentosa and Coats' disease. Archives of Ophthalmology (Chicago), 79, 146-149.

Notting, J. G. A., and Deutman, A. F. (1976). Leakage from retinal capillaries in hereditary dystrophies. In International Symposium on Fluorescein Angiography, Ghent 1976. Documenta Ophthalmologica Proceedings Series, vol. 9, pp. 439-447. Edited by J. J. De Laey. Junk: The Hague.

Notting, J. G. A., and Pinckers, A. J. L. G. (1977). Dominant cystoid macular dystrophy. American Journal of Ophthalmology, 83, 234-241.
Payne, J. W., and Patz, A. (1972). Treatment of acute proliferative retrolental fibroplasia. Transactions of the American Academy of Ophthalmology and Otolaryngology, 76, 1234-1246.

Pinckers, A., Deutman, A. F., and Notting, J. G. A. (1976). Retinal functions in dominant cystoid macular dystrophy (DCMD). Acta Ophthalmologica, 54, 579-590.

Schmidt, D., and Faulborn, J. (1970). Retinopathia pigmentosa mit Coats-Syndrom (Retinitis pigmentosa with Coats's syndrome). Klinische Monatsblätter für Augenheilkunde, 157, 643-652.

Schmidt, D., and Faulborn, J. (1972). Familiäres Vorkommen von Coats-syndrom kombiniert mit retinopathia pigmentosa. Klinische Monatsblätter für Augenheilkunde, 160, 158-163.

Spitznas, M., Joussen, F., Wessing, A., and MeyerSchwickerath, G. (1975). Coats's disease. An epidemiologic and fluorescein angiographic study. Albrecht von Graefes Archiv für Ophthalmologie, 195, 241-250.

Thijssen, J. M., Pinckers, A., and Otto, A. J. (1974). A multipurpose optical system for ophthalmic electrodiagnosis. Ophthalmologica (Basel), 168, 308-314.

Witschel, H. (1974). Retinopathia pigmentosa und 'Morbus Coats'. Klinische monatsblätter für Augenheilkunde, 164, 405-411.

Woods, A. C., and Duke, J. R. (1963). Coats's disease. I. Review of the literature, diagnostic criteria, clinical findings, and plasma lipid studies. British Journal of Ophthalmology, 47, 385-412.

Zamorani, G. (1956). Una rara associazione di retinite di Coats con retinite pigmentosa. Giornale italiano $d i$ oftalmologia, 2, 429-443. 